\title{
Estimation of Curvature along Curves with Application to Fibres in 3D Images of Paper
}

\author{
David Coeurjolly ${ }^{1}$ and Stina Svensson ${ }^{2}$ \\ 1 Laboratoire LIRIS \\ Université Lumère Lyon 2, Lyon, France \\ dcoeurjo@univ-lyon2.fr \\ 2 Centre for Image Analysis \\ Swedish University of Agricultural Sciences, Uppsala, Sweden \\ stina@cb.uu.se
}

\begin{abstract}
Space curves can be used to represent elongated objects in $3 \mathrm{D}$ images and furthermore to facilitate the computation of shape measures for the represented objects. In our specific application (fibres in 3D images of paper), we want to analyze the fibre network on a voxel level with respect to elongation and bending. For this purpose, it is of interest to estimate the curvature in each voxel along a curve. We present a new discrete curvature estimator fulfilling the multigrid convergence property. Moreover, we present results from a study where curvature together with other shape measures are used to analyze the fibre network.
\end{abstract}

\section{Introduction}

The 3D structure of fibres in paper is complex. To better understand its effect on the optical and mechanical properties of the paper, shape analysis of the fibres, performed from the 3D data, is required. Due to the rapid development within imaging, it is nowadays possible to generate $3 \mathrm{D}$ images of paper. This gives the opportunity to study the 3D structure of the fibres in a non-destructive way using computerized image analysis. It is pointed out in [1] that variation in fibre quality with respect to length, width, and shape are large and important for the industrial papermaking process.

So far, most methods to analyze fibres in papers based on the use of computerized image analysis have been developed for 2D (digital) images, or for 3D images but analyzed slice by slice, both resulting in the use of methods for $2 \mathrm{D}$ images. In [1], 2D images of dissolved paper samples are used. This is an efficient approach with respect to the number of fibres possible to analyze per time unit (10000 fibres in a few minutes). Many shape measures of the fibres can be extracted. However, all are based on planar shape, i.e., what can be seen in a 2D image.

In [2], the image acquisition technique used to obtain a 3D image of paper is described as well as a method to identify the individual fibres in the image. The 3D image resulting is of interest to use as an example of what can actually be said about the 3D structure of the fibres. This is a new research area within 
paper and pulp industry. Methods for computing some relevant measures are presented in $[3,4]$.

One shape measure of interest to study is the curvature along the fibre as it gives information on how the fibre is bending. This is relevant not only for fibres in paper. In [5], the curvature of wool fibres is suggested as a measure of crimp. In [6], the curvature of curve representations along fibres in unidirectional glassfibre-reinforced polymer composite are, together with other orientation related measure, used for representation of misalignments in the composites.

In this paper, we present a new method for estimating discrete curvature of a space curve in a 3D image (so far described only in the $\mathrm{PhD}$ thesis of Coeurjolly [7]). The method is an improvement of the method presented in [8] and has the multigrid convergence property. We use the curvature estimator, together with other measures, in a study on the fibres in a $3 \mathrm{D}$ image of paper. Each fibre is represented by a curve using the representation scheme presented in [4]. A preliminary study (on synthetic data only) of fibres using curvature as a shape measure can be found in [9].

\section{On fibres in 3D images of paper}

By StoraEnso Research in Falun, we have access to 3D data of paper. As a sample material, an uncoated milk cartoon was used. This type of duplex board contains a mixture of mechanical and chemical pulps which makes it interesting for analysis. In the image, the fibre walls, lumens, and void regions can be identified. The lumen is approximately cylindrically-shaped and, generally, the wall is a tube enclosing the lumen. The paper is captured slicewise using Scanning Electron Microscopy (SEM) and a sectioning device (a microtome). In each slice, the $2 \mathrm{D}(x, y)$-plane, a pixel is $0.7 \mu m \times 0.7 \mu m$ and the distance between each slice is $5.0 \mu \mathrm{m}$. Most fibres are aligned in one direction $(z)$ which is the machine direction during the papermaking process. This implies that in each $2 \mathrm{D}$ slice a fibre wall, which gives the highest respons using SEM, often has a ring-shaped form. The registration needed to create the $3 \mathrm{D}$ image is robust, due to the use of landmarks. More details can be found in [2]. An image sample is shown in Fig. 1.

We consider 3D images consisting of the fibre wall, the fibre lumen and void regions. We assume cubic voxels, i.e., the image described is resampled to cubic voxels. Each fibre is represented by a curve. Details on how the curve representation is computed can be found in [4]. The curve is centred within the fibre with the first (last) voxel in the curve (end-point) placed in the first (last) slice in which the fibre is present. Each voxel has exactly two neighbours in the curve except for the end-points. The curve representation of the fibre facilitates the measuring, such as length and curvature estimations, along the fibre. 


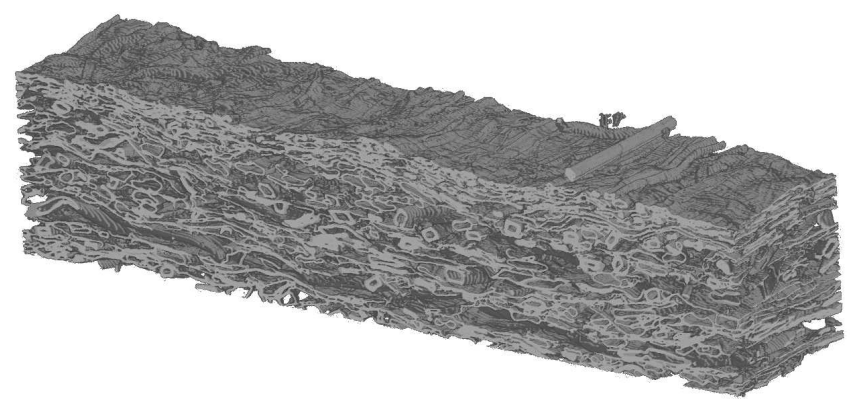

Fig. 1. A surface rendered sample of uncoated milk cartoon.

\section{A discrete curvature estimator}

We can consider a 26-connected space curve in a 3D digital image as a set of $m$ voxels $x_{0}, \ldots x_{m-1}$ for which each successive pair $\left\langle x_{i}, x_{i+1}\right\rangle$ is 26-connected to each other, i.e., $x_{i} \in N^{26}\left(x_{i+1}\right)$. The objective is to estimate the curvature at each point $x_{i}$ of this discrete curve. In [10], a first solution is presented for $n$-dimensional curves in grey-values images. However, discrete solutions are preferred as our original data is discrete.

First of all, we consider a Euclidean curve in 3D with a curvilinear abscissa parametrization $x(s)$. We have three classical ways to define the curvature of a curve or a path $x(s)$. The first one is based on the norm of the second derivative of the curve $\left(\kappa(s)=\left\|x^{\prime \prime}(s)\right\|\right)$. In $2 \mathrm{D}$ and $3 \mathrm{D}$, we can define a unitary tangent vector $\boldsymbol{t}(s)$ at each point and thus the curvature is given by the deviation of the curve according to the tangential line $\left(\kappa(s)=\left|\frac{d \boldsymbol{t}}{d s}\right|\right)$. Finally, we have a geometrical approach of the curvature definition given by the inverse of the osculating circle radius $r(s)\left(\kappa(s)=\frac{1}{r(s)}\right)$. In a continuous space, all these definitions are obviously equivalent whereas in discrete space, they lead to specific algorithms. Hence, several discrete estimators exist to compute the curvature at each point of a $2 \mathrm{D}$ discrete curve $[11,12,8]$. However, few algorithms can be extended to 3D digital curves.

In the PhD thesis of Coeurjolly [7], an algorithm to compute the curvature of a 3D discrete curve was proposed. We here present the algorithm (in English). It follows an approach that can be seen as a generalization of the classical order $m$ normalized curvature. Given a window size $m$ and a point $p_{i}$ of a $2 \mathrm{D}$ discrete curve, the estimated curvature $\kappa^{*}\left(p_{i}\right)$ is given by ${ }^{3}$ :

$$
\kappa^{*}\left(p_{i}\right)=\frac{\angle\left(p_{i-m} p_{i}, p_{i} p_{i+m}\right)}{\left|p_{i-m} p_{i}\right|+\left|p_{i} p_{i+m}\right|}
$$

To generalize this simple estimator, we consider the two half-tangents for each voxel $v_{0}=\left(x_{0}, y_{0}, z_{0}\right)$, i.e., the discrete straight line segments starting in

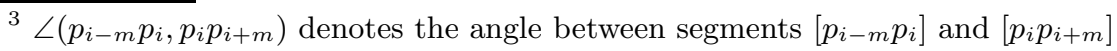


$v_{0}$ in the direction of the two end-points of the curve, respectively. In that case, the parameter $m$ is adapted for each half-tangent according to the geometry of the curve. Let us denote the end-points of the discrete straight line segments by $v_{1}=\left(x_{1}, y_{1}, z_{1}\right)$ and $v_{2}=\left(x_{2}, y_{2}, z_{2}\right)$. We estimate the osculating circle at the point $v_{0}$ of the discrete curve as the circumcircle of the triangle $\left(v_{0}, v_{1}, v_{2}\right)$. To compute the radius for the circumcircle, we can directly use the formula in [13]: let $a$ be the Euclidean distance from $v_{0}$ to $v_{1}, b$ the Euclidean distance from $v_{1}$ to $v_{2}, c$ the Euclidean distance from $v_{0}$ to $v_{2}$, and $\mathcal{A}$ the area of the triangle $\left(v_{0}, v_{1}, v_{2}\right)$. Then we have that

$$
\mathcal{A}=\frac{\sqrt{(b+c)^{2}-a^{2}} \cdot \sqrt{a^{2}-(b-c)^{2}}}{4.0}
$$

The curvature in $v_{0}$ is given by the (inverse of the) radius of the circumscribing circle of the $3 \mathrm{D}$ triangle $\left(v_{0}, v_{1}, v_{2}\right)$, i.e., $R_{c}=a b c / 4 \mathcal{A}$ (see Fig. 2 ).



Fig. 2. Illustration, in the $2 \mathrm{D}$ case, of the half-tangent approach for the curvature estimation.

In [7], we proved that the curvature estimator based on discrete half-tangents fulfil the multigrid convergence property. In other words, if we consider a Euclidean curve $x(s)$ and a digitization process of $x(s)$ up to a certain grid resolution, the curvature estimation on the digitization of $x(s)$ converges to the exact Euclidean value when the grid resolution increases. There, also an algorithm to compute half-tangents at each voxel in optimal time, i.e., in a linear time with respect to the number of voxels of the curve, was presented. Hence, the described algorithm estimates the curvature at each voxel in a discrete curve in a linear time.

\section{Measurements on fibres in an uncoated milkcartoon}

From the 3D image of paper, we have used 17 fibres. In fact, as described in [2], the process to identify individual fibres is far from trivial. We use some of the 
fibres that have been properly identified to illustrate our methods. For each fibre, we computed the curve representation, [4]. Guided by the curve a number of measures is extracted. The aim is to give a description on how the fibre is behaving in the 3D space with respect to elongation and bending.

In Fig. 3, we show projections of one fibre, left, and its curve representation, right, from the sample in Fig. 1.
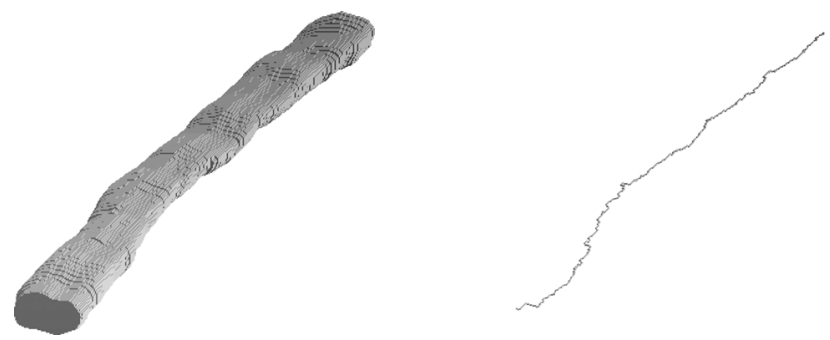

Fig. 3. One fibre, left, and its curve representation, right.

The measure in focus for this paper is the estimation of curvature $(\kappa)$ along the fibre. It is of interest to give the mean curvature for each fibre as well as the variance of the curvature. High variation in curvature is likely to indicate strong interactions between neighbouring fibres or non-rigid fibre walls. In fact, this type of fibre-to-fibre bond is frequent in paper and is what makes the paper "stay together". Each bonding contributes to the overall strength of the paper.

As pointed out in Section 2, most fibres are aligned along one direction $(z)$. To have an indication on how well aligned they actually are, we give their extensions in $x$-, $y$-, and $z$-direction $(\Delta x, \Delta y, \Delta z)$.

An often used measure is fibre curl $(C)$, which is equal to the length of the fibre divided by the length of a straight line connecting its end-points. Fibre curl measures the crookedness of the fibre, which has shown to be of importance in many contexts, [1]. To compute the length $(L)$ of the fibre, we use the length estimator presented in [14]. It is based on the idea to segment the curve into straight line segments and to estimate the total length using the length of the segments. The algorithm for identifying the straight line segments is the same as the one used to compute the half-tangents needed to estimate the curvature.

In Table 1, the results for the 17 fibres are presented.

\section{Discussion}

In this paper, we present a new discrete curvature estimator and apply it in a study of the fibre network in 3D images of paper.

From Table 1, we see that, as suspected, the fibres are mostly aligned along the $z$-direction $(\Delta z \gg \Delta x, \Delta y)$. We also see that the fibres, on average, are more slanted in the $x$-direction than in the $y$-direction $(\Delta x>\Delta y)$. In fact, during 
Table 1. Shape measures for fibres. Length is given in $\mu m$.

\begin{tabular}{r|rrrrccc} 
fibre & \multicolumn{1}{c}{$L$} & \multicolumn{1}{c}{$\Delta x$} & $\Delta y$ & \multicolumn{1}{c}{$\Delta z$} & $C$ & $\operatorname{mean}(\kappa)$ & $\operatorname{var}(\kappa)$ \\
\hline 1 & 538.21 & 7.0 & 10.5 & 504.0 & 1.07 & 0.053 & 0.005 \\
2 & 536.10 & 84.7 & 11.2 & 504.0 & 1.05 & 0.072 & 0.006 \\
3 & 562.56 & 142.8 & 23.8 & 504.0 & 1.07 & 0.064 & 0.006 \\
4 & 442.40 & 123.2 & 2.1 & 388.5 & 1.09 & 0.125 & 0.012 \\
5 & 546.04 & 43.4 & 21.7 & 504.0 & 1.08 & 0.074 & 0.007 \\
6 & 540.83 & 10.5 & 4.2 & 504.0 & 1.07 & 0.063 & 0.006 \\
7 & 574.91 & 143.5 & 23.1 & 504.0 & 1.10 & 0.061 & 0.005 \\
8 & 187.81 & 63.7 & 2.1 & 149.1 & 1.16 & 0.099 & 0.012 \\
9 & 564.61 & 21.7 & 4.2 & 504.0 & 1.12 & 0.069 & 0.007 \\
10 & 431.04 & 32.2 & 7.0 & 383.6 & 1.12 & 0.086 & 0.010 \\
11 & 267.42 & 4.9 & 18.9 & 254.1 & 1.05 & 0.067 & 0.006 \\
12 & 288.74 & 56.7 & 1.4 & 254.1 & 1.11 & 0.098 & 0.010 \\
13 & 234.89 & 133.7 & 48.3 & 119.0 & 1.27 & 0.149 & 0.013 \\
14 & 571.85 & 92.4 & 9.8 & 499.1 & 1.13 & 0.088 & 0.012 \\
15 & 87.91 & 30.1 & 1.4 & 68.6 & 1.17 & 0.106 & 0.009 \\
16 & 581.34 & 83.3 & 4.2 & 504.0 & 1.14 & 0.107 & 0.009 \\
17 & 216.34 & 44.8 & 16.1 & 193.9 & 1.08 & 0.070 & 0.007
\end{tabular}

manufacturing of paper, the pulp is subjected to strong forces which causes the fibres to aggregate in clusters essentially aligned along $2 \mathrm{D}$ planes $((x, z)$-planes in this case). This layered structure makes the fibre position less restricted in the $x$-direction, i.e., within such a plane, than in $y$-direction, i.e., between different such planes.

From the fibre curl measure $C$, we see that most fibres are rather straight as $C$ is close to 1 . If $C \equiv 1$, the curve representation coincides with a straight line between its two end-points. Values of $C$ significantly larger than 1 indicates deviation from a straight line.

High values of $C$ does not necessarily implies high curvature $\kappa$ (neither the mean nor the variance). For example, this does not hold for fibre 9 for which mean and variance of $\kappa$ is low while $C$ is high. In fact, the table shows that $C$ and $\kappa$ do not give the same information but are both useful to describe the general bending of a fibre as well as fast changes in it. Using $\kappa$, we can have information on how the fibre deviates from a straight line. If the variance of $\kappa$ is low, the mean of $\kappa$ gives an indication of the frequency in the "waviness" of the fibre.

In our data set, most fibres are well behaved, in the sense that $C$ and $\kappa$ are close to 1 and low, respectively. The fibres with higher values are often short. In this case, the high values can be related to approximations necessary in the discretization process more than to the actual shape of the fibre.

Future work will be to investigate in detail how $\kappa$ changes along the fibre and how much this is related to the fibre-to-fibre bond areas. This is an interesting feature compared to $C$ which gives one value for the whole fibre. Most likely, $\kappa$ is low in correspondence with fibre-to-fibre bond areas, as the fibres are more 
fixed also in the $x$-direction. However, studies on a larger image in which more fibres have been identified is necessary to verify this.

Our original data is discrete. For this reason, we have chosen to use a purely discrete approach, both for extracting the curve representation used for each fibre and for measuring shape properties from it. By a discrete approach, we can obtain voxel by voxel measurements using the only information we have, i.e., the information given by the discrete $3 \mathrm{D}$ image, and this can be done in a computationally convenient way.

\section{Acknowledgements}

StoraEnso Research, Falun, Sweden is acknowledged for providing the original image data.

Dr Mattias Aronsson, Centre for Image Analysis, Uppsala, Sweden is gratefully acknowledged for scientific support and for the work with preprocessing the $3 \mathrm{D}$ image with fibres, from image registration to identification of individual fibres, used in this study.

\section{References}

1. Karlsson, H., Fransson, P.I.: STFI FiberMaster gives the papermaker new muscles - New knowledge about fiber shape can be the key to tomorrow's paper. Internet. http://www.stfi.se/documents/contract/pulp/fibermart.htm (2000) Accessed January 15, 2003.

2. Aronsson, M.: On 3D Fibre Measurements of Digitized Paper. PhD thesis, Swedish University of Agricultural Sciences (2002) Acta Universitatis Agriculturae Sueciae. Silvestria 254 .

3. Aronsson, M., Svensson, S.: Curvature measurements for fibres in 3D images of paper. In Åström, K., ed.: Proceedings SSAB'02 Symposium on Image Analysis, Lund, Sweden (2002) 165-168

4. Svensson, S., Aronsson, M.: Using distance transform based algorithms for extracting measures of the fibre network in volume images of paper. Accepted for publication in IEEE Transactions on System, Man and Cybernetics. Special issue on 3D Image Analysis and Modeling (2003)

5. Sommerville, P.: Fibre curvature - What is all the fuss about? Internet. http://www . awta.com.au/Publications/Fact_Sheets/Fact_sheet_004.htm (2000) Accessed January 15, 2003.

6. Clarke, A., Eberhardt, C.: The representation of reinforcing fibres in composites as 3D curves. Composites Science and Technology 59 (1999) 1227-1237

7. Coeurjolly, D.: Algorithmique et géométrie discrète pour la caractérisation des courbes et des surfaces. PhD thesis, Université Lumière Lyon 2, Bron (2002)

8. Coeurjolly, D., Miguet, S., Tougne, L.: Discrete curvature based on osculating circle estimation. In Arcelli, C., Cordella, L.P., Sanniti di Baja, G., eds.: Visual Form 2001. Volume 2059 of Lecture Notes in Computer Science., Springer-Verlag (2001) 303-312

9. Svensson, S.: Detecting a $D^{6}$ surface skeleton using iterative thinning. In Bigün, J., Malmqvist, K., eds.: Proceedings Symposium on Image Analysis (SSAB'00), Halmstad, Sweden, Halmstad University (2000) 37-40 
10. Rieger, B., van Vliet, L.J.: Curvature of $n$-dimensional space curves in grey-value images. IEEE Transactions on Image Processing 11 (2002) 738-745

11. Worring, M., Smeulders, A.W.M.: Digital curvature estimation. Computer Vision, Graphics, and Image Processing. Image Understanding 58 (1993) 366-382

12. Vialard, A.: Geometrical parameters extraction from discrete paths. In: 6th Discrete Geometry for Computer Imagery. Volume 1176 of Lecture Notes in Computer Science., Springer-Verlag (1996) 24-35

13. Harris, J.W., Stocker, H.: Handbook of mathematics and computational science. Springer-Verlag (1998)

14. Coeurjolly, D., Debled-Rennesson, I., Teytaud, O.: Segmentation and length estimation of 3D discrete curves. In Bertrand, G., Imiya, A., Klette, R., eds.: Digital and Image Geometry. Volume 2243 of Lecture Notes in Computer Science., Dagstuhl, Germany, Springer-Verlag (2002) 299-317 